\title{
INVARIANTS UNDER DECOMPOSITION OF THE CONJUGATION IN THE MOD 2 DUAL LEIBNIZ-HOPF ALGEBRA
}

\author{
NEŞET DENIZ TURGAY
}

Received 10 April, 2018

\begin{abstract}
The Leibniz-Hopf algebra is the free associative algebra on one generator, $S^{n}$, in each positive degree, with coproduct $\Delta\left(S^{n}\right)=\sum S^{j} \otimes S^{n-j}$. Let $\mathcal{C}$ and $\mathcal{R}$ denote coarsening and reversing operations on the mod 2 dual Leibniz-Hopf algebra. We consider decomposition of the Hopf algebra conjugation $\chi=\bigodot \circ \mathcal{R}$ in this dual Hopf algebra and calculate bases for the fixed points of the operations $\mathcal{C}$ and $\mathcal{R}$.
\end{abstract}

2010 Mathematics Subject Classification: 16T05; 57T05; 55S10

Keywords: Hopf algebra, Leibniz-Hopf algebra, antipode, Steenrod algebra, quasisymmetric functions

\section{INTRODUCTION}

The Leibniz-Hopf algebra $\mathcal{F}$ is the free associative $\mathbf{Z}$-algebra on generators $S^{1}, S^{2}, S^{3} \ldots$ with the graded cocommutative Hopf algebra structure determined by $\Delta\left(S^{n}\right)=\sum_{i+j=n} S^{i} \otimes S^{j}$ (where $S^{0}$ denotes the unit 1 ). $\mathcal{F}$ is isomoprhic to the 'ring of noncommutative symmetric functions' [7] and has been studied in [9-11]. The graded dual Hopf algebra, $\mathscr{F}^{*}=\oplus_{n} \operatorname{Hom}\left(\mathcal{F}_{n}, \mathbf{Z}\right.$ ) (where $\mathscr{F}_{n}$ denotes the degree $n$ part of $\mathscr{F}$ ), is the ring of quasi-symmetric functions with the outer coproduct, which has been studied in $[2,6,8-12]$. This algebra was the subject of the Ditters conjecture $[1,5,11]$ which makes it important in combinatorics. The mod 2 reduction $\mathscr{F} \otimes \mathbf{Z} / 2$ also has a connection with topology, since the mod 2 Steenrod algebra is naturally defined as a quotient of $\mathcal{F} \otimes \mathbf{Z} / 2$ by the Adem relations [13]. From now on we denote $\mathcal{F} \otimes \mathbf{Z} / 2$ by $\mathcal{F}_{2}$.

As $\widetilde{F}_{2}$ is the free $\mathbf{Z} / 2$-algebra on $S^{1}, S^{2}, \ldots$, a basis for $\mathscr{F}_{2}$ is given by all words $S^{j_{1}} S^{j_{2}} \ldots S^{j_{l}}$. We denote the corresponding dual basis for $\mathscr{F}_{2}^{*}=\oplus_{n} \operatorname{Hom}\left(\mathcal{F}_{n}, \mathbf{Z} / 2\right)$ by $\left\{S_{j_{1}, j_{2}, \ldots, j_{l}}\right\}$. Since $\mathcal{F}$ is the cocommutative graded Hopf algebra, $\mathcal{F}^{*}$ is a commutative graded Hopf algebra with a unique conjugation operation, $\chi$, which satisfies 
$\chi^{2}=1$ (where 1 denotes the identity homomorphism). A formula for $\chi$ was introduced by Ehrenborg [6, Proposition 3.4] and for $\mathcal{F}_{2}{ }^{*}$ we can simplify it to:

$$
\chi\left(S_{j_{1}, j_{2}, \ldots, j_{l}}\right)=\sum S_{i_{1}, \ldots, i_{k}}
$$

summed over all coarsenings $i_{1}, \ldots, i_{k}$ of the reversed word $j_{l}, \ldots, j_{1}$, i.e., all words $i_{1}, \ldots, i_{k}$ that admit $j_{l}, \ldots, j_{1}$ as a refinement. As an example,

$$
\chi\left(S_{5,2,1}\right)=S_{1,2,5}+S_{3,5}+S_{1,7}+S_{8} .
$$

The length of a word is the number of its letters so $S_{2,1,2,6}$ has length 4 . A word of length $n$ has $n-1$ commas and, hence, $2^{n-1}$ coarsenings.

Using conjugation invariants in $\mathcal{F}_{2}{ }^{*}$ is an algebraic tool to understand the conjugation invariants in the mod 2 dual Steenrod algebra [14, Section 5]. Motivated by this in [4], the author and Crossley calculated a vector space basis for the invariants of $\widetilde{F}_{2}^{*}$ under the operation $\chi$. In [3] the analogous question was considered for $\widetilde{F}_{2}$. In [16, Section 6] the author and Kaji introduced an explicit correspondence between the invariant elements of $\mathcal{F}_{2}$ and of $\mathcal{F}_{2}{ }^{*}$ under the Hopf algebra conjugation operation. See also [15] for the relationship between the conjugation invariants in $\mathscr{F}_{2}$ and the conjugation invariants in the mod 2 Steenrod algebra.

The conjugation operation $\chi$ comprises both coarsening and reversing operations. In this paper we calculate vector space bases for the invariants under coarsening and reversing operations. We also investigate relations between those spaces with the space of conjugation invariants in $\mathcal{F}_{2}^{*}$.

\section{TERMinOLOGY AND RESULTS}

Given a word $S_{i_{1}, i_{2}, \ldots, i_{k}}$, define its image under the map $\mathcal{C}$ to be

$$
\varphi\left(S_{i_{1}, i_{2}, \ldots, i_{k}}\right)=\sum S_{l_{1}, \ldots, l_{n}}
$$

summed over all coarsenings $l_{1}, \ldots, l_{n}$ of $i_{1}, \ldots, i_{k}$, and define its image under the map $\mathcal{R}$ to be $\mathcal{R}\left(S_{i_{1}, i_{2}, \ldots, i_{k}}\right)=S_{i_{k}, \ldots, i_{2}, i_{1}}$. Here, $\mathcal{C}$ and $\mathcal{R}$ denote coarsening and reversing operations on $\mathscr{F}_{2}^{*}$. It is clear that $\mathcal{R}^{2}=1$. Both $\mathcal{C}$ and $\mathcal{R}$ are homomorphisms on $\mathcal{F}_{2}^{*}$, and by definition we have: $\chi=\mathcal{C} \circ \mathcal{R}$. Moreover, one can see that $\mathscr{C}$ and $\mathcal{R}$ commute, i.e., $\mathcal{C} \circ \mathcal{R}=\mathcal{R} \circ \mathcal{C}$. This together with the property that $\chi^{2}=1$ implies that $\ell^{2}=1$.

An element $x \in \mathcal{F}_{2}^{*}$ is an invariant under $\mathcal{C}$ if, and only if, $\mathcal{C}(x)=x$, i.e., ( $\mathcal{C}-$ 1) $(x)=0$. Hence, $\operatorname{Ker}(\mathcal{C}-1)$ is a subspace of $\mathcal{F}_{2}^{*}$, which is formed by the invariants under $\mathcal{C}$ in $\mathcal{F}_{2}{ }_{2}$. Similarly, $\operatorname{Ker}(\mathcal{R}-1)$ is a subspace of $\mathcal{F}_{2}{ }^{*}$, which is formed by the invariants under $\mathcal{R}$ in $\mathcal{F}_{2}{ }_{2}$. We now introduce a new terminology. A word $S_{i_{1}, i_{2}, \ldots, i_{k}}$ is said to be uniterminal if its last letter is equal to 1 , i.e., $i_{k}=1$. We denote this word by UT.

As an example, in the degree 4 part of $\mathcal{F}_{2}^{*}$ the UTs are: $S_{3,1}, S_{2,1,1}, S_{1,2,1}, S_{1,1,1,1}$. We also recall terminologies from [4]. A word $S_{i_{1}, i_{2}, \ldots, i_{k}}$ is a palindrome if $i_{1}=i_{k}$, 
$i_{2}=i_{k-1}$, etc. The non-palindromes form obvious pairs: a non-palindrome $S_{i_{1}, \ldots, i_{k}}$ pairs with $S_{i_{k}, \ldots, i_{1}}$. In each pair, one term will be higher in lexicographic ordering, and one lower. We call the first an HNP (higher non-palindrome) and the second an LNP. For example, $S_{5,4,1,3}$ is an HNP, $S_{4,1,2,4}$ is an LNP.

Theorem 1. In the mod 2 dual Leibniz-Hopf algebra invariants under $C$ comprise exactly the image of $\mathcal{C}-1$, i.e., $\operatorname{Ker}(\mathcal{C}-1)=\operatorname{Im}(\mathcal{C}-1)$.

Theorem 2. In degree $n$ part of mod 2 dual Leibniz-Hopf algebra the subspace $\operatorname{Im}(\mathcal{R}-1)$ has a basis consisting of the $(\mathcal{R}-1)$-images of all HNPs.

Consequently the dimension of $\operatorname{Im}(\mathcal{R}-1)$ in degree $n$ is $2^{n-2}-2^{(n-2) / 2}$ if $n$ is even, and $2^{n-2}-2^{(n-3) / 2}$ if $n$ is odd.

The dimension results in the last statement are obtained by simple combinatorial calculations. (See [4, Section 3] and [3, Section 2] for details). Furthermore, Theorem 2 together with the rank and nullity theorem gives the result.

Theorem 3. Let $\operatorname{Ker}(\mathcal{R}-1)_{n}$ denote the subspace of degree $n$ invariants under $\mathcal{R}$ in the mod 2 dual Leibniz-Hopf algebra. Then

$$
\operatorname{dim} \operatorname{Ker}(\mathcal{R}-1)_{n}=\left\{\begin{array}{l}
2^{n-2}+2^{(n-2) / 2}, \text { if } n \text { is even, } \\
2^{n-2}+2^{(n-3) / 2}, \text { if } n \text { is odd. }
\end{array}\right.
$$

Theorem 4. The space of invariants under $\mathcal{R}, \operatorname{Ker}(\mathcal{R}-1)$, has a basis consisting of the following:

(1) All the palindromes; and

(2) The $(\mathcal{R}-1)$-images of all HNPs.

Proposition 1. In the mod 2 dual Leibniz-Hopf algebra we have:

(1) $\operatorname{Ker}(\chi-1) \cap \operatorname{Ker}(\mathcal{C}-1)=\operatorname{Ker}(\chi-1) \cap \operatorname{Ker}(\mathcal{C}-1) \cap \operatorname{Ker}(\mathcal{R}-1)$.

(2) $\operatorname{Ker}(\chi-1) \cap \operatorname{Ker}(\mathcal{R}-1)=\operatorname{Ker}(\chi-1) \cap \operatorname{Ker}(\mathcal{R}-1) \cap \operatorname{Ker}(\mathcal{C}-1)$.

(3) $\operatorname{Ker}(\mathcal{C}-1) \cap \operatorname{Ker}(\mathcal{R}-1)=\operatorname{Ker}(\mathcal{C}-1) \cap \operatorname{Ker}(\mathcal{R}-1) \cap \operatorname{Ker}(\chi-1)$.

\section{Proof of Theorem 1}

We first give the following auxiliary results.

Theorem 5. The image of $(\mathcal{C}-1)$ on $\mathcal{F}_{2}^{*}$ has a basis consisting of the $(\mathcal{C}-1)$ images of all UTs.

Lemma 1. Let $S_{i_{1}, \ldots, i_{k}}$ be a UT. Among the summands of longest length in (e 1) $\left(S_{i_{1}, \ldots, i_{k}}\right)$ there is a summand $S_{i_{1}, \ldots, i_{k-2}, i_{k-1}+i_{k}}$, and this summand does not occur as a longest length summand in the $e-1$ image of any other UT.

Proof. Let $S_{i_{1}, \ldots, i_{k}}$ be a $k$ length UT, then the longest summands in $(\mathcal{C}-1)\left(S_{i_{1}, \ldots, i_{k}}\right)$ are of length $k-1$, and $S_{i_{1}, \ldots, i_{k-2}, i_{k-1}+i_{k}}$ is one of them. We shall show that $S_{i_{1}, \ldots, i_{k-2}, i_{k-1}+i_{k}}$ cannot arise as a longest summand in the $(\mathcal{C}-1)$ image of any 
other UT, say $S_{j_{1}, \ldots, j_{l}}$. In $(\mathcal{C}-1)\left(S_{j_{1}, \ldots, j_{l}}\right)$, similarly the longest summands are of length $l-1$, namely,

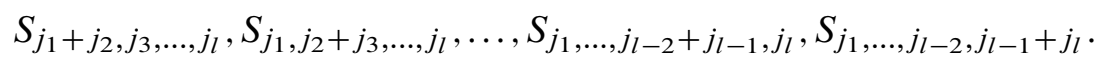

We see that all the terms in (3.1) are UTs except the last term $S_{j_{1}, \ldots, j_{l-2}, j_{l-1}+j_{l}}$ $\left(j_{l-1}+j_{l}>1\right)$. For this term to equal $S_{i_{1}, \ldots, i_{k-2}, i_{k-1}+i_{k}}$, we must have $l=k,, j_{1}=$ $i_{1}, j_{2}=i_{2}, \ldots, j_{l-2}=i_{k-2}$, and $j_{l-1}+j_{l}=i_{k-1}+i_{k}$. By definition, $j_{l}=i_{k}=1$ which implies that $S_{j_{1}, \ldots, j_{l}}=S_{i_{1}, \ldots, i_{k}}$. On the other hand, none of the UT terms in (3.1) can equal to a non-UT term $S_{i_{1}, \ldots, i_{k-2}, i_{k-1}+i_{k}}$. This completes the proof.

Proof of Theorem 5. Suppose that we have a sum of UTs whose image under $\ell-1$ is 0 . Order these summands so that shorter terms come before longer terms. Let $S_{i_{1}, \ldots, i_{k}}$ be the last summand with respect to this ordering, then it has the term $S_{i_{1}, \ldots, i_{k-2}, i_{k-1}+i_{k}}$ as longest summand in its image under $\mathcal{C}-1$. Lemma 1 tells us that having length $k-1$, this term cannot arise in the $(\mathcal{C}-1)$-image of any shorter term or of any other UT of length $k$. Hence, in the $(\mathcal{C}-1)$-image of the sum the term $S_{i_{1}, \ldots, i_{k-2}, i_{k-1}+i_{k}}$ cannot be cancelled, so this image cannot be zero. This contradicts the hypothesis showing that $S_{i_{1}, \ldots, i_{k}}$ cannot be a UT. This implies that the sum is itself zero. Hence, the UTs have linearly independent images under $\ell-1$.

The number of UTs in degree $n$ is $2^{n-2}$, since each UT has 1 as the last term and the remaining terms can be any word of degree $n-1$, of which there are $2^{n-2}$. Hence, in $n$ degrees, the UTs form a set of $2^{n-2}$ elements. Thus, the above linear independence establishes that $\operatorname{dim} \operatorname{Im}(e-1) \geq 2^{n-2}$. On the other hand, $\operatorname{Im}(e-1) \subset$ $\operatorname{Ker}(\mathcal{C}-1)$, since $\mathcal{C}^{2}=1$, so in each degree we have $\operatorname{dim} \operatorname{Im}(\mathcal{C}-1) \leq \frac{1}{2} \operatorname{dim}\left(\mathcal{F}_{2}{ }^{*}\right)$. In all degrees, this means $\operatorname{dim} \operatorname{Im}(\mathcal{C}-1) \leq \frac{1}{2} 2^{n-1}=2^{n-2}$. Consequently, $\operatorname{dim} \operatorname{Im}(\mathcal{C}-$ $1)=2^{n-2}$. We know the $(e-1)$-images of UTs are linearly independent and the number of UTs matches $\operatorname{dim} \operatorname{Im}(C-1)$. Hence, the $(\complement-1)$-images of UTs must be a basis for $\operatorname{Im}(e-1)$. Moreover, this shows that $\operatorname{Ker}(e-1)=\operatorname{Im}(e-1)$.

This completes the proof of Theorem 1 giving a basis for $\operatorname{Ker}(\mathcal{C}-1)$.

\section{Proof of Theorem 4}

We first deal with Theorem 2 (Theorem 3 was proved in Section 2).

Proof of Theorem 2. Let $S_{i_{1}, \ldots, i_{k}}$ be an HNP, then it is clear that the longest summands in $(\mathcal{R}-1)\left(S_{i_{1}, \ldots, i_{k}}\right)$ are $S_{i_{1}, \ldots, i_{k}}$ and its reverse. It is clear that these summands cannot arise in the $(\mathcal{R}-1)$-image of any other HNP. Using this fact together with the argument in the proof of Theorem 5 shows that the $(\mathcal{R}-1)$-images of all HNPs are linearly independent. What is left to show that no other terms contribute anything further to the image. i.e., the $(\mathcal{R}-1)$-image of every palindrome and LNP can be expressed in terms of the ( $\mathcal{R}-1)$-images of HNPs. Let $S_{i_{1}, \ldots, i_{k}}$ be a palindrome, then $(\mathcal{R}-1)\left(S_{i_{1}, \ldots, i_{k}}\right)=0$. On the other hand, if $S_{i_{1}, \ldots, i_{k}}$ be an LNP, then 
$(\mathcal{R}-1)\left(S_{i_{1}, \ldots, i_{k}}\right)=(\mathcal{R}-1)\left(S_{i_{k}, \ldots, i_{1}}\right)$, where $S_{i_{k}, \ldots, i_{1}}$ is a HNP. Hence, the image of $(\mathcal{R}-1)$ on $\mathcal{F}_{2}^{*}$ is as stated.

Lemma 2. In the mod 2 dual Leibniz-Hopf algebra all the palindromes and the $(\mathcal{R}-1)$-images of all HNPs are linearly independent.

Proof. Suppose that there are distinct palindromes $p_{1}, \ldots, p_{k}$ and there are distinct HNPs $h_{1}, \ldots, h_{r}$ such that

$$
p_{1}+\cdots+p_{k}=(\mathcal{R}-1)\left(h_{1}\right)+\cdots+(\mathcal{R}-1)\left(h_{r}\right) .
$$

The longest summands (i.e., the maximal-length summands) on the left of Eq. (4.1) are all palindromes and these summands cannot cancel, since $p_{1}, \ldots, p_{k}$ are different. On the other hand, the longest summands in $(\mathcal{R}-1)\left(h_{j}\right)$ are $h_{j}$ and its reverse, both of which are non-palindromes. Again, none of these summands can cancel since $h_{1}, \ldots, h_{r}$ are all different HNPs. Thus the maximal-length summands on the right of Eq.(4.1) are non-palindromes. This contradiction establishes that Eq.(4.1) can only hold if both sides are 0 . Hence all the palindromes and the $(\mathcal{R}-1)$-images of all HNPs are linearly independent.

By Lemma 2 all the palindromes and the $(\mathcal{R}-1)$-images of all HNPs are linearly independent. We will complete the proof of Theorem 4 by using the dimension argument that we used for the proof of Theorem 5. Elementary combinatorial calculations shows us that the number of palindromes in degree $n$ is $2^{n / 2}$ if $n$ is even, and $2^{(n-1) / 2}$ if $n$ is odd. (See [4, Section 3] for details). By Theorem 2 we also know the number of HNPs in even and odd degrees. From this we can see the number of palindromes and HNPs in each degree matches the dimension given in Theorem 3. Hence, all the palindromes and the $(\mathcal{R}-1)$-images of all HNPs form a basis.

Proof of Proposition 1. We prove (1), the proofs of (2) and (3) being similar. Let $x \in \operatorname{Ker}(\chi-1) \cap \operatorname{Ker}(\mathcal{C}-1)$, then $\chi(x)=x$ and $\mathscr{C}(x)=x$. This implies that $\chi(x)=x=(\mathcal{R} \circ \mathcal{C})(x)=\mathcal{R}(x)$ from which we can deduce $\operatorname{Ker}(\chi-1) \cap \operatorname{Ker}(\mathcal{C}-$ 1) $\subset \operatorname{Ker}(\mathcal{R}-1)$. This completes the proof.

\section{Acknowledgements}

I wish to thank MD Crossley and S. Kaji for their comments on the earlier version of this paper.

\section{REFERENCES}

[1] M. D. Crossley, "The Steenrod algebra and other copolynomial Hopf algebras." Bull. Lond. Math. Soc., vol. 32, no. 5, pp. 609-614, 2000, doi: 10.1112/S0024609300007128.

[2] M. D. Crossley, "Some Hopf algebras of words." Glasg. Math. J., vol. 48, no. 3, pp. 575-582, 2006, doi: 10.1017/S0017089506003302. 
[3] M. Crossley and N. D. Turgay, "Conjugation invariants in the Leibniz-Hopf algebra." J. Pure Appl. Algebra, vol. 217, no. 12, pp. 2247-2254, 2013, doi: 10.1016/j.jpaa.2013.03.003.

[4] M. Crossley and N. D. Turgay, "Conjugation invariants in the mod 2 dual Leibniz-Hopf algebra." Commun. Algebra, vol. 41, no. 9, pp. 3261-3266, 2013, doi: 10.1080/00927872.2012.682675.

[5] E. J. Ditters, "Curves and formal (co) groups." Invent. Math., vol. 17, pp. 1-20, 1972, doi: 10.1007/BF01390019.

[6] R. Ehrenborg, "On posets and Hopf algebras.” Adv. Math., vol. 119, no. 1, pp. 1-25, 1996, doi: 10.1006/aima.1996.0026.

[7] I. M. Gelfand, D. Krob, A. Lascoux, B. Leclerc, V. S. Retakh, and J.-Y. Thibon, "Noncommutative symmetric functions." Adv. Math., vol. 112, no. 2, pp. 218-348, 1995, doi: 10.1006/aima.1995.1032.

[8] M. Hazewinkel, "Generalized overlapping shuffle algebras.” J. Math. Sci., New York, vol. 106, no. 4, pp. 3168-3186, 2001, doi: 10.1023/A:1011386821910.

[9] M. Hazewinkel, "The algebra of quasi-symmetric functions is free over the integers." Adv. Math., vol. 164, no. 2, pp. 283-300, 2001, doi: 10.1006/aima.2001.2017.

[10] M. Hazewinkel, "Symmetric functions, noncommutative symmetric functions, and quasisymmetric functions." Acta Appl. Math., vol. 75, no. 1-3, pp. 55-83, 2003, doi: 10.1023/A:1022323609001.

[11] M. Hazewinkel, "Explicit polynomial generators for the ring of quasisymmetric functions over the integers.” Acta Appl. Math., vol. 109, no. 1, pp. 39-44, 2010, doi: 10.1007/s10440-009-9439-z.

[12] C. Malvenuto and C. Reutenauer, "Duality between quasi-symmetric functions and the Solomon descent algebra." J. Algebra, vol. 177, no. 3, pp. 967-982, 1995, doi: 10.1006/jabr.1995.1336.

[13] J. W. Milnor, "The Steenrod algebra and its dual.” Ann. Math. (2), vol. 67, pp. 150-171, 1958, doi: $10.2307 / 1969932$.

[14] N. D. Turgay, "An alternative approach to the Adem relations in the mod 2 Steenrod algebra." Turk. J. Math., vol. 38, no. 5, pp. 924-934, 2014, doi: 10.3906/mat-1309-6.

[15] N. D. Turgay, "A remark on the conjugation in the Steenrod algebra." Commun. Korean Math. Soc., vol. 30, no. 3, pp. 269-276, 2015, doi: 10.4134/CKMS.2015.30.3.269.

[16] N. D. Turgay and S. Kaji, "The mod 2 dual Steenrod algebra as a subalgebra of the mod 2 dual Leibniz-Hopf algebra." J. Homotopy Relat. Struct., vol. 12, no. 3, pp. 727-739, 2017, doi: 10.1007/s40062-016-0163-x.

\section{Author's address}

\section{Neşet Deniz Turgay}

Department of Mathematics, Faculty of Arts and Sciences, Eastern Mediterranean University, Famagusta, North Cyprus, Via Mersin 10, Turkey

E-mail address: neset.turgay@emu.edu.tr 